\title{
A Mutual Selection Mechanism of Ride-Hailing Based on Hidden Points
}

\author{
Yi Jiang $\mathbb{C}^{1,2}$ Yu Xia, ${ }^{1}$ Xinyue Cheng, ${ }^{1}$ and Yuntao $\mathrm{Xu}^{1}$ \\ ${ }^{1}$ School of Information Engineering, Yangzhou University, Yangzhou Jiangsu 225127, China \\ ${ }^{2}$ State Key Laboratory of Ocean Engineering, Shanghai Jiao Tong University, Shanghai 200240, China \\ Correspondence should be addressed to Yi Jiang; jiangyi@yzu.edu.cn
}

Received 20 February 2020; Revised 28 May 2020; Accepted 18 June 2020; Published 15 July 2020

Academic Editor: Huimin Lu Copyright ( $2020 \mathrm{Yi}$ Jiang et al. This is an open access article distributed under the Creative Commons Attribution License, which
permits unrestricted use, distribution, and reproduction in any medium, provided the original work is properly cited.

\begin{abstract}
As a new way to travel, ride-hailing has brought great convenience to the public. However, the demand expression ability of passengers in the existing ride-hailing platforms is weak, and the accuracy of the matching results is low, resulting in a large number of transaction losses and the loss of platform revenue. In this paper, we propose a kind of mutual selection mechanism of ride-hailing based on hidden points, which is composed of platform benefit calculation algorithm and driver assignment algorithm. The platform benefit calculation algorithm mainly calculates the benefits obtained by the platform after the order is completed, while the driver allocation algorithm meets the mutual selection needs of passengers and drivers. Through experiments and theoretical analysis, the mutual selection mechanism proposed in this paper has improved user satisfaction by 5\%-21\% compared to the traditional methods of order-snatching mechanism and order-sending mechanism, which has significantly improved passenger satisfaction and solved the problem that the existing platform cannot meet the passengers' individual travel needs and the problem that the passengers' choice is not fair. The issue of platform revenue was discussed in the context of improved passenger satisfaction.
\end{abstract}

\section{Introduction}

With the continuous development of modern cities, the public has higher and higher requirements for the convenience, rapidity, and comfort of transportation. In densely populated large cities, public transportation such as buses and subways sometimes cannot meet the special needs of the public. At this time, ride-hailing platforms emerge as the times require, which greatly satisfied the diverse travel needs of the public, greatly improved people's travel efficiency, and also provided more jobs. After several years of development, ride-hailing has become one of the first choices for the public to travel. Different ride-hailing platforms will adopt different matching rules, and different matching rules will lead to differences in platform matching results. The main purpose of the traditional ride-hailing platform is to improve the matching efficiency and complete more matching in a shorter time. It is reflected that the passenger side is to match the passengers in the shortest possible time. The traditional ride-hailing platform is a bipartite matching problem. In this problem, a bipartite graph $G=(U, V, E)$ can be presented, where $U$ represents the online driver and $V$ represents the passenger's order request. If $v$ is assigned to $u$, there is an edge $e=(u, v)$. When a passenger's order request $v$ arrives, the ride-hailing platform must make a decision to reject the request or assign $v$ to $u$.

Currently, the typical ride-hailing platforms are Didi and Uber, which adopt different matching rules. The Didi platform will push the orders submitted by users to the drivers around the starting point, and the drivers will grab the orders, and the drivers who grab the orders will become the sellers of the orders. This pattern matching rule is relatively simple, and time efficiency is high, but there are some problems with this pattern: the engineer is in a state of tight time to scramble for orders, and travel safety cannot be guaranteed. In addition, malicious order grabbing and order canceling will also reduce the satisfaction of drivers and passengers, which will eventually lead to lower revenue of the platform. Unlike Didi, Uber uses a dispatch model: through a series of complex matching rules, the one who meets the needs of 
customers' order sent to a matching degree is the highest driver, the driver needs to respond within a certain amount of time, and if the driver did not respond or refuse orders, the platform will order to other drivers, until a driver receives the order. Under this matching rule, Uber needs to take certain punitive measures to make drivers cautious about receiving orders and improve responsiveness. However, due to the existence of punishment measures, the number of online drivers will be smaller than that of Didi, and the ultimate matching efficiency will be lower than that of Didi.

Another problem with the existing matching rules is the problem of selection fairness. The existing matching rules mainly allow the driver to decide whether to accept or snatch the order, and the platform determines the final matching result. Passengers just issue orders without much choice. The advantage of this matching rule is that it reduces intermediate links and effectively guarantees the matching efficiency. However, the matching rule will also deprive passengers of the right to choose. Drivers can choose passengers while passengers cannot choose drivers, which is part of the reason for the recent vicious incidents of online ridehailing. To some extent, the fairness of choice affects users' satisfaction with drivers and ride-hailing platforms.

In order to solve the problem of user satisfaction and complexity of matching rules caused by the fairness of selection, this paper proposes a mutual selection mechanism based on hidden points. This paper is divided into six chapters: the second section introduces the related work. The third section introduces the model and the details of mechanism. The fourth section introduces the algorithm and analysis of the mechanism. The fifth section introduces the experiment part. The final section summarizes the full text. The introduction should be succinct, with no subheadings. Limited figures may be included only if they are truly introductory and contain no new results.

\section{Related Work}

Compared with the traditional way of travel, online ridehailing has certain advantages. Chen et al. [1] proposed a heuristic algorithm, which showed that ride-sharing is an effective method to reduce the number of vehicles required for travel and vehicle mileage. In particular, when the degree of participation is high and the starting point and destination of the journey are more spatially concentrated, carpooling can reduce more vehicle miles traveled and the number of vehicles used. Ride-hailing platforms also need to have a strategy to attract potential users, so that the operation of the platform enters a virtuous cycle. Wang et al. [2] studied the factors influencing potential users to use online ridehailing platforms. Henao and Marshall [3] analyzed the relevant data of drivers and concluded that after completing the order, drivers should stop and wait for the next ride request instead of driving to the active area, unless doing so can reduce the ride request waiting time by at least $30 \%$. Based on the SOR model, Yang and Chen [4] constructed a factor model of copassenger satisfaction and analyzed customer satisfaction from four dimensions: perceived usefulness, perceived ease of use, perceived travel risk, and perceived service quality. Gilibert et al. [5] contributed to the new DRT mechanism by identifying user needs and market opportunities. Segal-Halevi et al. [6] proposed a real multiunit bilateral auction mechanism, in which, by dividing the market into left and right submarkets, eventually, the mechanism are prior-free, dominant-strategy incentive-compatible, individually rational, and budget-balanced. Lee et al. [7] proposed a taxi dispatching system based on real-time traffic conditions. Miao et al. [8] combined data information with real-time control decision to balance the minimum total free driving distance between an idle taxi and the minimum free driving distance. By analyzing Uber's system architecture, Watanabe et al. [9] found the importance of price decline and order increase in a virtuous cycle to the platform architecture. $\mathrm{Xu}$ et al. [10] proposed an order scheduling algorithm based on a large-scale car-hailing platform, aiming to provide a more effective method for optimizing resource utilization and user experience from a global and longerterm perspective and significantly improve the allocation efficiency of the platform. Henao and Marsall [11] analyzed the impact of ride-hailing on traffic efficiency and concluded that the vehicle mileage brought by ride-hailing was much higher than that without ride-hailing. Pham et al. [12] enable privacy-conscious riders to achieve levels of privacy that are not possible in the current RHSs and even in some conventional taxi services, thereby offering a potential business differentiator. Young and Farber [13] show that ride-hailing is too minute and inconsequential to influence the ridership level of other more substantial modes of travel overall, when considering specific market segments; the rise of ride-hailing corresponds to a significant decrease in taxi ridership and a rise in active modes of travel. And it will have a much more pronounced effect on the level of ridership of other modes as well. de Souza Silva et al. [14] show that the majority of ridesourcing trips is replacing taxi and public transport trips. Safety and cost are the main reasons that influence the decision of sharing trips via ride-splitting. The use of larger vehicles for sharing trips can introduce competition with the public transport systems. The ridesourcing interference on collective public transportation may be more noticeable than on individual public transport (taxis), given the much greater demand for the former. Mäntymäki et al. [15] say the stark power disparity between workers and the platform is, in turn, a major source of discontent among workers and put forward two key dimensions of work relations in the context of platform-enabled work: digital temporality and algorithmic administration. Guo et al. [16] assess the impact of ride-hailing platforms' market entry on new car purchases in the presence of platform competition and found the two competing platforms may have provided subsidies to drivers such that more people purchased new cars in order to sign up as drivers. Ma et al. [17] report on the development of an integrated model to investigate how perceptions of risk play into a person's decision to stop using a particular ride-hailing service and users' trust in drivers has a positive effect on users' trust in the ride service platform and their attitude towards the platform; users' trust in the platform positively affects their attitude towards the platform. 
In general, most of the relevant studies focus on platforms and drivers, with demand forecasting and vehicle scheduling as the focus [18-21]. However, few studies have addressed the problem of passenger satisfaction from the perspective of passengers. The passenger satisfaction largely determines the retention rate of users. If the problem of user satisfaction cannot be solved well, the platform will lose users and eventually lead to lower revenue. Therefore, this paper focuses on the research of passenger satisfaction and proposes a mutual selection mechanism for ride-hailing based on hidden points. This mechanism improves the effectiveness of the platform on the basis of solving passenger satisfaction.

\section{Model and Mechanism Design}

3.1. Model. There are three main roles in the ride-hailing matching mechanism: driver $(u)$, passenger $(v)$, and ridehailing platform. A driver-owned vehicle can be seen as a commodity that can take passengers to their destination. As the provider of services, the driver can act as a seller in the market. Passengers issue demands and provide money. As the party buying services, passengers issue service requests to ride-hailing platforms and provide fees after the driver sends them to their destination, which can be understood as the buyer in the market. The ride-hailing platform provides a price function, matches drivers and passengers, and charges a certain handling fee as revenue. Both drivers and passengers are users of the platform. As the party providing the platform, the ride-hailing platform can be understood as a market maker in the market (see Figure 1).

Each user $i$ in the matching mechanism has its own value function $v_{i}$, which is initialized to $v_{i}(0)=0$. The user's value function is determined by the benefits brought by the trip. The value function of the driver is the cost and profit composition of the service. In this paper, it is assumed that the platform has $n$ orders, the price function set by the platform is $p$, and the travel distance is $m$ in an order $j(j \in[0, n])$. So for buyer $v, \operatorname{Gain}_{j, v}(m, p)=v_{v}(m)-p(m)$. For seller $u$, $\operatorname{Gain}_{j, u}(m, p)=p(m)-v_{u}(m)-h . h$ represents the no-load cost of the driver. This distance is only cost and no benefit.

The matching mechanism generates a matching result $G=(U, V, E)$ and a transaction price function $p$ according to the passenger's demand order. In the matching mechanism proposed in this paper, the matching result is generated by the mutual selection of the driver and the passenger. Both of them need to accept the matching result and be responsible for the matching result and pay a certain transaction fee to the platform $f_{j}$, where $f_{j}=f_{u}+f_{v}$. If a matching mechanism is budget balanced, then $\sum_{j \in \mathrm{Order}} f_{j} \geq 0$. If each user receives a positive return, then a mechanism is personally rational; that is, $\operatorname{Gain}_{j}(m, p)-f_{j} \geq 0$ is inevitable. If a user cannot increase his own income by falsely reporting the valuation, then, this mechanism is real. This paper defines the passenger satisfaction function as $s_{j}$. User satisfaction will bring some hidden benefits to the platform. Then Utility $=\sum_{j=1}^{n} f_{j}+s_{j}-C$, where $C$ represents platform operating costs.
3.2. Mechanism Details. Under this mechanism, passengers send a service request order to the online ride-hailing platform at time $t$ to request service. Based on the principle of proximity, the online ride-hailing platform first uses the hidden point matching mechanism to first select the $X$ drivers closest to the user's departure point, then filter out $m$ drivers close to the user's hidden points, order will be pushed to the $m$ drivers, $n$ drivers out of $m$ drivers $(n \leq m)$, then the platform pushes the information of the $n$ drivers (gender, distance, vehicle condition, vehicle type, driving speed, etc.) to the user, and the user selects the service driver. At this time, the driver and passenger are successfully matched, that is, the value $e=(u, v)$. The driver is a reusable resource [22]. The driver grabs orders at time $t^{\prime}$, and the passenger decides to serve the driver at time $t^{\prime \prime}$.The order is completed at time $t^{\prime \prime \prime}$, then the driver will rejoin the matching queue after time $t^{\prime \prime \prime}$, and the passenger will occupy the time $t^{\prime \prime \prime}-t$, which is the service time. The occupancy time of driver $u$ matching with passenger $v$ depends on the user type (such as destination) of $v$ and the time when the matching occurs (peak time may be significantly different from off-peak time).

Hidden points' mechanism using weighted algorithm calculates hidden points for driver and passenger separately. Hidden points for drivers include the order grabbing rate, order completion rate, user evaluation, and complaint rate. The order grabbing rate refers to the driver's acceptance rate of orders pushed by the platform. Order completion rate refers to the proportion of the number of orders grabbed by the driver and completed by the driver in the number of orders grabbed by the driver. The order grabbing rate and order completion rate will combine to affect the hidden score of the driver. User evaluation includes multiple scores, including passenger satisfaction with service, driver's service attitude, vehicle environment, vehicle speed, and whether to arrive on time, and a comprehensive evaluation score is obtained through a weighting algorithm. Both the driver and the platform have the right to view user evaluations. Drivers can improve their service quality based on user evaluations, provide better services, improve their hidden points, and obtain higher profits. The complained rate refers to the proportion of the driver's complained orders in the number of orders he completed. The complaint rate is proportional to the hidden ratio. The passenger's complaint against the driver will be handled manually by the online car-hailing platform. The passenger's evaluation of the platform will be reflected in the user satisfaction later. Passenger hidden score includes complaint rate, usage rate, and driver evaluation. Among them, the complaint rate refers to the proportion of passenger complaint orders in all the submitted orders. When users encounter some problems that are difficult to coordinate with the driver, they can use complaints to protect their rights and interests. Under normal circumstances, the passenger's complaint rate will be maintained at a normal level, but if the passenger's complaint rate exceeds the normal level, it will be classified as a poor-quality passenger, and the complaint rate is inversely proportional to the hidden share. Utilization rate refers to the frequency with which passengers use the ride-hailing platform, and the utilization rate 


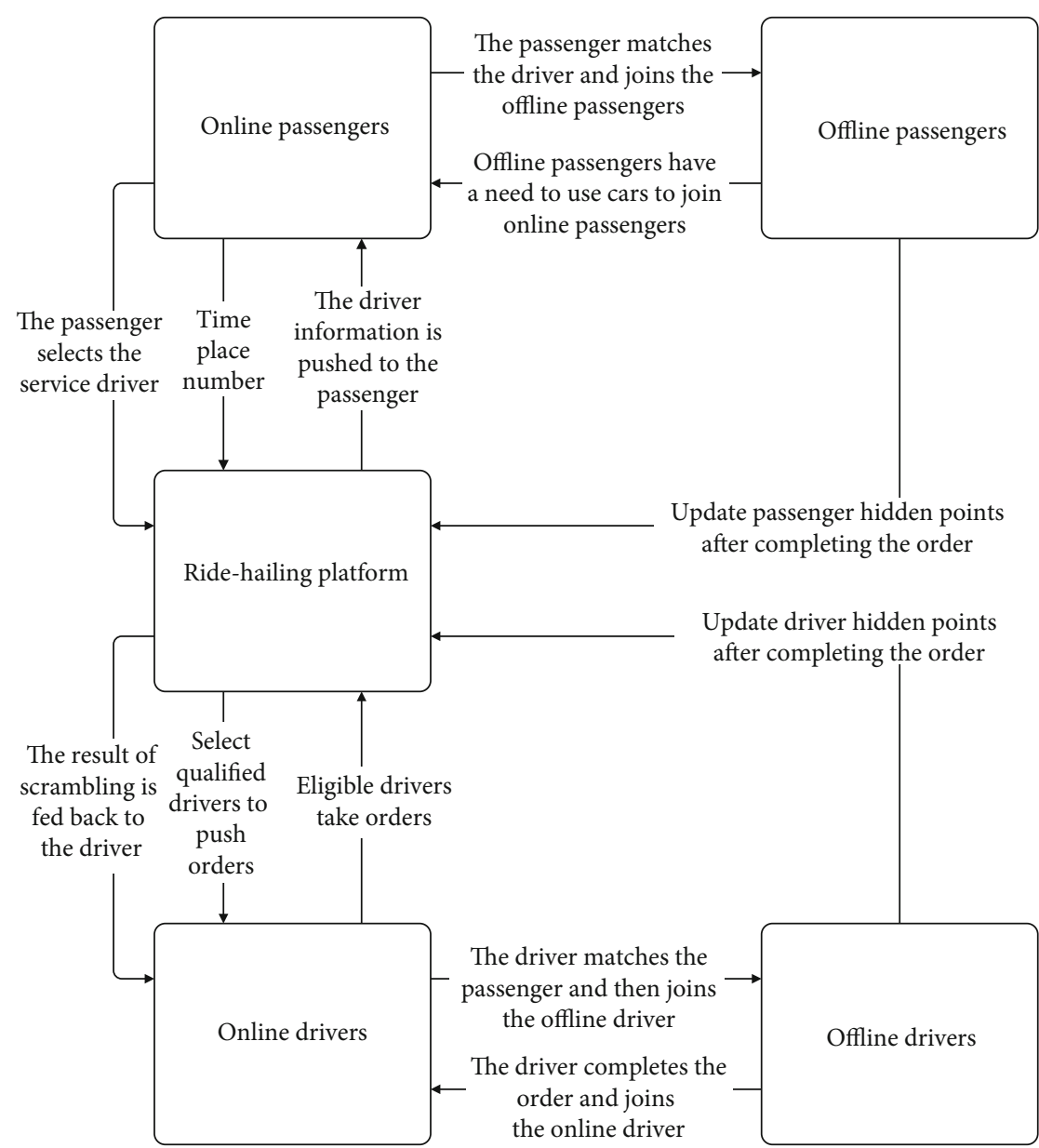

FIGURE 1: Structure diagram of ride-hailing matching.

evaluation is proportional to the concealment. Driver evaluation refers to the driver's evaluation of passengers. The higher the evaluation, the higher the hidden score. Drivers and passengers evaluate each other. The evaluation is not displayed in real time (that is, it can be evaluated after the order is completed), but it can be evaluated one minute after the order is completed, and the evaluation content is not displayed in real time, but only after the mutual evaluation of both parties is seen. This reduces false ratings to a certain extent, because under the scoring mechanism of the existing platform, the order can be evaluated once the order is completed, and some drivers will require passengers to give a full score evaluation, even if some passengers are not satisfied with the itinerary, only a full score evaluation. Delaying the evaluation time can to a certain extent ensure that passengers leave the driver's visible range for evaluation, which improves the authenticity of the evaluation.

(i) Hidden points for drivers:

$$
\mathrm{HP}_{d_{i}}=\sum_{k \in[K] n=1,2} \sum_{i}^{n} w_{k^{n}} \frac{1}{k_{i}^{3}} k_{i}^{4},
$$

where for drivers and $K$ is the order scrambling rate, order receiving completion rate, complained rate, and user evaluation. (ii) Hidden points for passengers:

$$
\mathrm{HP}_{p_{i}}=\mathrm{k}_{i}^{1} \frac{1}{k_{i}^{2}} k_{i}^{3}
$$

where for passengers, $K$ is the usage rate, complaint rate, and driver evaluation.

In this paper, assuming the driver's Supply for Supply ${ }_{u}$, and the Demand of users for Demand $\mathrm{d}_{v}$, generally speaking, we have excess demand ( Demand $_{v}>$ Supply $_{u}$ ), excess supply (Demand ${ }_{v}<$ Supply $_{u}$ ), and Supply and Demand which are equal (Demand ${ }_{v}=$ Supply $_{u}$ ). In the ride-hailing platform, when Demand ${ }_{v}>$ Supply $_{u}$, it means that it is the current time to peak, and when Demand ${ }_{v}<$ Supply $_{u}$, it means that the current session is a low period. In this paper, we only discuss the case of oversupply, where the number of drivers far outweighs the number of user orders.

The step is shown Figure 2.

Step 1. Passengers send a service request to the platform, platform passenger departure, arrival, classification of hidden information to be obtained, and then on passenger source, screening from the starting point of the recent $X$ driver, according to the $X$ driver hidden points; filter out $m$ drivers 


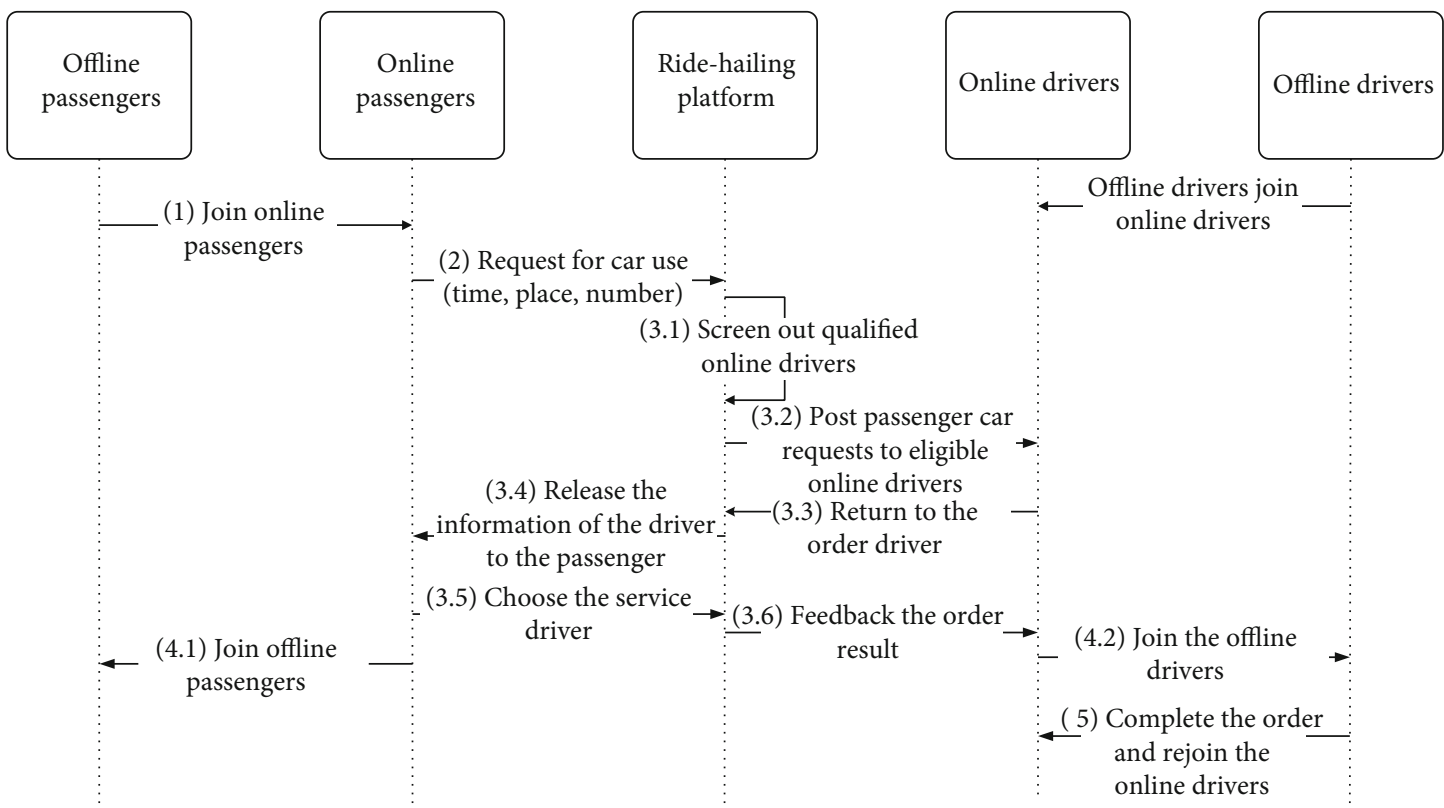

Figure 2: Timing sequence diagram of ride-hailing matching.

who are close to the user's hidden points; and then push user orders to these $m$ drivers.

Step 2. Among the $m$ drivers, there will be $n$ drivers scrambling for orders $(n \leq m)$, and then the platform will push the information of these $n$ drivers (gender, distance, vehicle condition, vehicle type, average driving speed, etc.) to the user, who will finally choose the service driver. After the passenger selects the service driver, the platform matches the two, adds the service driver to the offline pool, returns the information of the successful order snatcher to the driver, and returns the information to the $n-1$ order snatcher to inform them of the failed order snatcher.

Step 3. After the journey is completed, the platform calculates the fee that passengers should pay to the driver and the transaction fee $f_{j}$ that users need to pay to the platform based on the unit price $p$ of the mileage. The transaction fee $f_{j}$ is paid by the passenger and the driver, respectively. After the transaction is completed, the driver and passenger evaluate each other about the service, and the evaluation results are hidden from both parties on the day and visible to both parties on the following day.

3.3. Algorithm and Analysis. This section introduces a matching algorithm of driver-passenger mutual selection based on the hidden points, which is aimed at designing a matching rule. On the basis of ensuring the matching efficiency, the algorithm enables passengers to have certain right of demand expression, improves user satisfaction, and finally increases the revenue of the platform.

For each driver, there is a certain probability to decide whether to snatch the order. In this paper, the probability of the driver's decision to snatch the order is defined as $x_{e}$. For each passenger, each driver has a matching weight value; that is, matching $e=(u, v)$ is related to a weight $w_{e}$. Since the driver is a reusable resource, let $t^{\prime}<t$. In formula (4), the first term represents that $u$ is unavailable at time $t$, and the second term represents that $u$ was assigned to other passengers at time $t$. The probability that is less than $u$ is available at time $t$.

$$
\begin{gathered}
\max \sum_{t \in[T]} \sum_{e \in E} x_{e} w_{e} U t i \\
\text { subject to } 0 \leq x_{e} \leq 1 \forall e \in \mathrm{E}, \quad t \in[T], \\
\sum_{t^{\prime}<t} \sum_{e \in E_{u}} x_{e, t^{\prime}}+\sum_{e \in E_{u}} x_{e, t} \leq 1 \forall u \in U, \quad t \in[T] .
\end{gathered}
$$

The distribution benefit algorithm of the ride-hailing platform is shown in Algorithm 1, where $\Theta_{t}$ represents the set of drivers in the driver pool waiting at time $t$. $U=\left\{u, g_{u}, S_{u}\right\}$ is the driver information set, where $u$ represents the number of the driver, $g_{u}$ represents the hidden score of the driver numbered with $u, S_{u}$ represents the state of the driver numbered with $u, S_{u}=1$ means the driver is online and waiting for orders, and $S_{u}=0$ means the driver is offline or carrying passengers. $\Omega_{j}\left\{l_{v}, \mathrm{t}, g_{v}, S_{j}\right\}_{i \in I}$ said orders $j$ collection of information, $l_{v}$ said user $v$ origin, $t$ time, $g_{v}$ said user $v$ hidden points, and $S_{j}$ said orders $j$ order status. The Utility $=\sum_{j=1}^{n} f_{j}+s_{j}-C$ represents the platform's revenue.

When passengers $v$ order submission requirements for platform, system at time $t \in\left[a_{j}, d_{j}\right]$ in the distribution of the driver to the user. The system allocates drivers through an allocation algorithm, as shown by Algorithm 2. After the order is completed, the platform needs to modify the status of the driver and update the hidden point information of the driver pool and calculate the revenue of the order. 


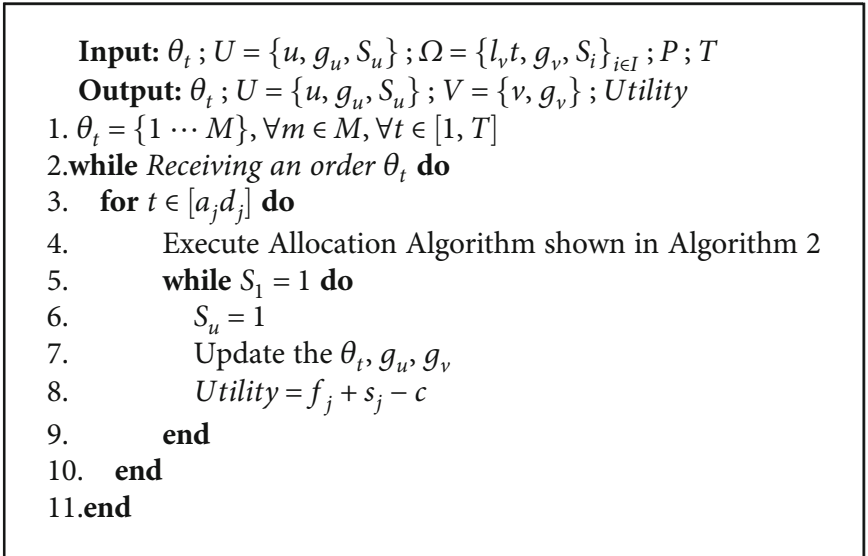

Algorithm 1: Online platform.

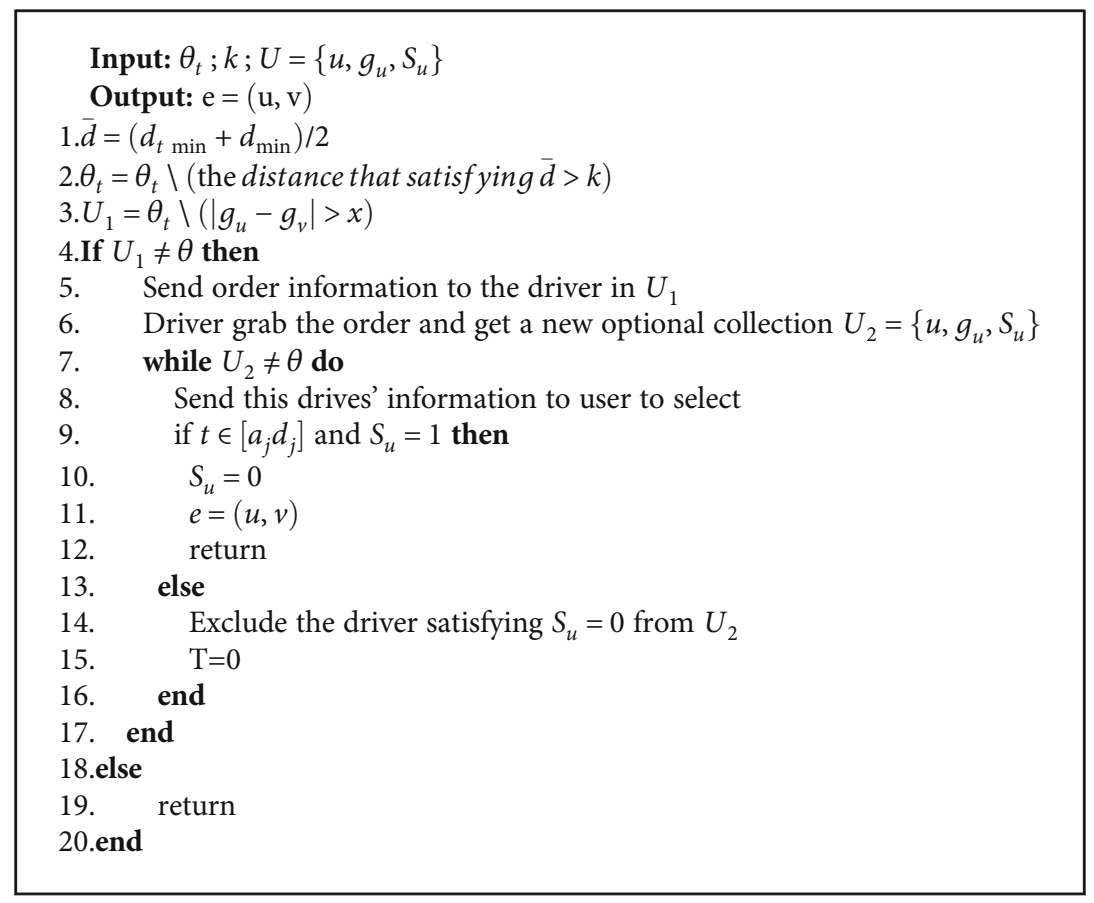

Algorithm 2: Allocation algorithm.

In Algorithm 2, $k$ represents the maximum screening distance between the driver and the passenger defined by the system during the primary election. According to the source information of users in the order, the system screened out all drivers whose distance from the source is less than $k$. Distance $\bar{d}$ was driver's fastest time average distance and the shortest distance. $e=(u, v)$ represents the successful pairing of the driver and passenger. $x$ is the score difference set by the system. In order to provide better service, the system will automatically match the score difference between passengers and drivers within $x$. There may be multiple passengers using ride-hailing services at the same time, and a driver may also meet the needs of multiple passengers. The allocation algorithm sets a timer, and the user must select the driver within the valid time to prevent the driver from being selected by other passengers or getting pulled off the line abnormally. Because of the double-option mechanism, the driver is likely to have been selected by user B before being selected by user A, which also encourages the user to choose as soon as possible. If the driver has been selected, the system pushes the set of drivers back to the user until the match is successful or there is no driver to choose from in the driver pool.

Theorem 1. The matching mechanism is prior-free, dominant-strategy, incentive-compatible, individually rational, and budget-balanced.

Proof. A priori free is determined by the mechanism, which does not need to collect users' value information. In dominant-strategy and incentive-compatible, for passengers, only by sending an order can they get the service. The behavior of sending an order is the dominant strategy, while for drivers, no matter how other drivers choose, the behavior of scrambling for an order is the dominant strategy. Under 
this mechanism, passengers and drivers are unable to increase their earnings by inflating the valuation. The price is set by the platform, and neither side can influence the pricing. Therefore, the mechanism is incentive compatible. Through the platform, passengers and drivers cooperate with each other and finally realize the maximization of their respective interests, which is obviously rational for individually rational. The mechanism is budget balanced; that is, the platform will not lose money and can obtain transaction fees from the transaction.

Theorem 2. Under this algorithm, the improvement of passenger satisfaction will bring more hidden benefits to the platform.

Proof. Under this mechanism, passengers' satisfaction will be enhanced due to their breeding needs being satisfied to a certain extent, and their own needs are attached importance to the platform, and the fairness of platform matching is guaranteed. The passengers' satisfaction will drive new passenger to join the platform, and the increasing of the number of passengers will make more drivers choose to use the platform, and an increase in the number of driver will attract more people to join platform into the benign circulation state, eventually rise in the number of users, and bring more benefits to the platform.

User satisfaction consists of three parts: matching time, matching efficiency, and evaluation,

$$
s_{j}=\sum_{k \in[K]} \sum_{n=1}^{3} L \frac{1}{\left(t^{\prime \prime}-t\right)} x_{e} w_{e} k^{n} w_{k^{n}},
$$

where $L$ is a constant and $K$ is the platform evaluation, service quality, and demand satisfaction.

3.4. Experiments. In order to verify the effectiveness of the mechanism proposed herein, this paper simulates a scenario in which there is a ride-hailing platform with 1 passenger and 20 drivers randomly distributed around passengers. The experiment simulates three different matching methods (platform A adopted the pattern of scrambling for orders, platform $\mathrm{B}$ adopted the pattern of dispatching orders, and platform $\mathrm{C}$ adopted the matching rules proposed in this paper) and finally generates 3 sets of data, each set of data with 50 records. The records generated by different matching rules are different. The simulation data of platform A contains the matching time of the order, driver's order grabbing rate, user evaluation, and other data; the data of platform $\mathrm{B}$ contains the order matching time, the driver's order acceptance rate, and the user evaluation and other data; the data simulated by platform $\mathrm{C}$ includes the matching time of orders, driver's rate of obtaining orders, user's consent rate, user's evaluation of platform, user's evaluation of itinerary, and user's demand satisfaction. The simulated data includes the matching time, matching efficiency, users' evaluation of the platform, users' evaluation of the journey, and users' satisfaction degree for each order under different rules.

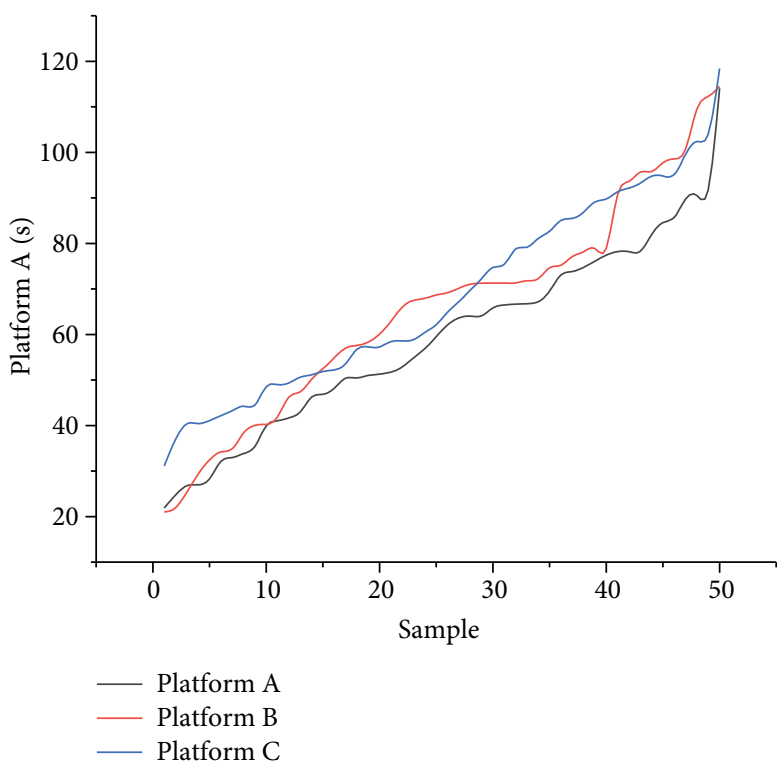

Figure 3: Comparison of platform matching time.

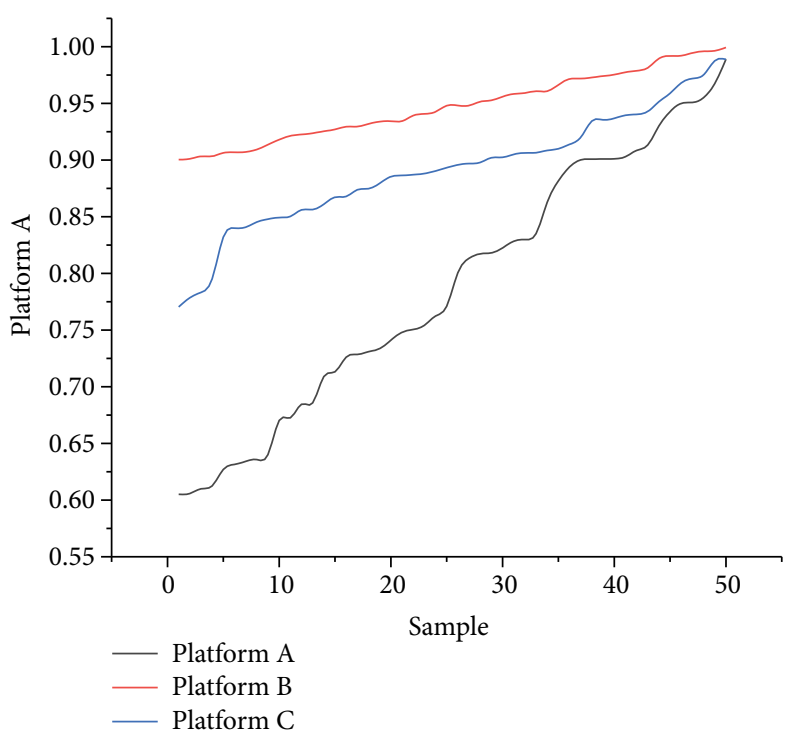

FIgURE 4: Comparison of platform matching rate.

This experiment assumes that under three platforms, the number of drivers is much larger than that of passengers, which ensures that the order matching efficiency under different platforms will not be affected by the relationship between supply and demand. Three different matching rules were used to calculate the satisfaction of each group of 50 data under each matching rule, and the experimental results were as follows.

From the above figure, we can see that the overall matching time of platform $\mathrm{C}$ is longer than those of platform $\mathrm{A}$ and platform $\mathrm{B}$. The average matching time of platform $\mathrm{C}$ is $15 \%$ longer than that of platform $A$ and 3.6\% longer than that of platform $B$ (see Figure 3). The overall matching rate of platform $\mathrm{C}$ is better than that of platform $\mathrm{A}$ and inferior to that of platform $\mathrm{B}$. The average matching rate of platform $\mathrm{C}$ is $6 \%$ lower than that of platform $B$ and $12 \%$ higher than that of platform A (see Figure 4). The overall evaluation of 


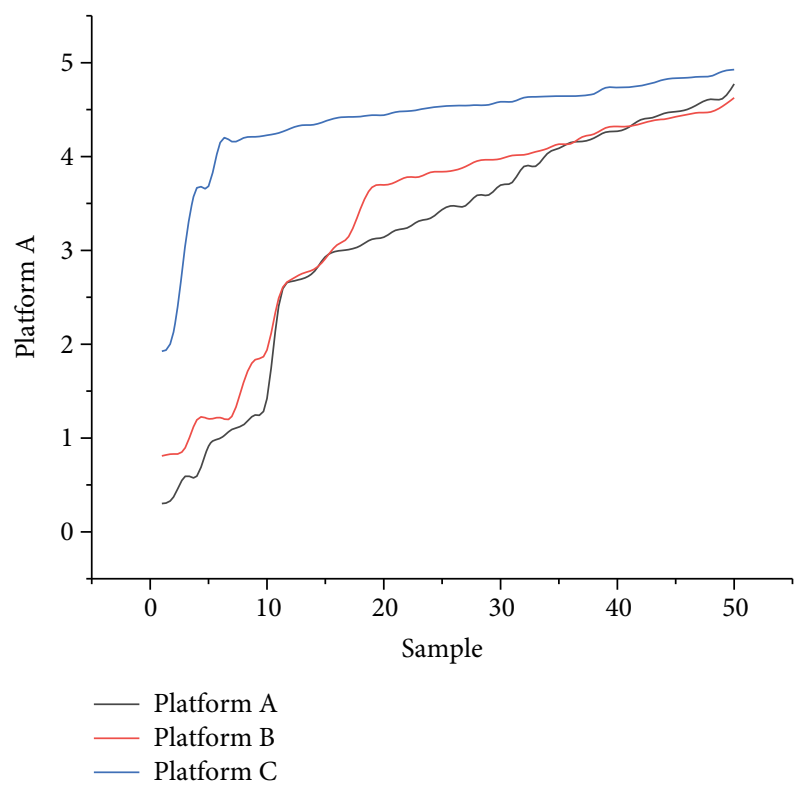

FIGURE 5: Comparison of platform evaluation.

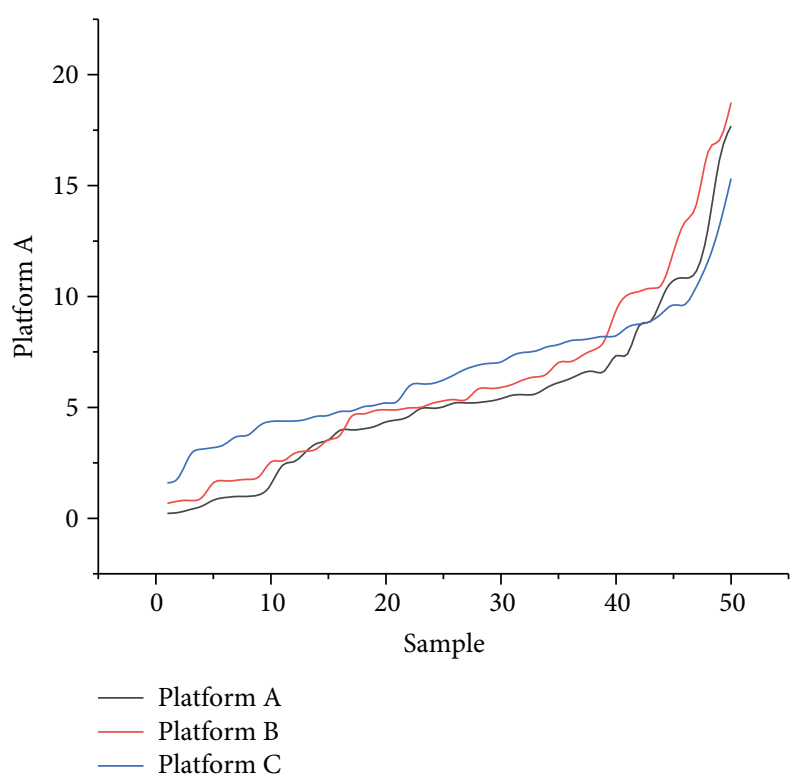

FIGURE 6: Comparison of platform satisfaction.

platform $\mathrm{C}$ is better than those of platform $\mathrm{A}$ and platform $\mathrm{B}$. The average evaluation of platform $C$ is $39 \%$ higher than that of platform A and 30\% higher than that of platform B (see Figure 5). The overall user satisfaction of platform $C$ is better than those of platform A and platform B. The satisfaction of platform $C$ is $21 \%$ higher than that of platform A and $5 \%$ higher than that of platform B (see Figure 6).

Since platform A adopts the order scrambling rule, the platform will send orders to 20 drivers, and 20 drivers will scramble the order. The competition ratio is $1 / 20$. Although the matching speed of platform $A$ has a great advantage over those of platforms $\mathrm{B}$ and $\mathrm{C}$, the final matching result is unpredictable and cannot reflect the expression of demand by passengers. The driver's competition is fierce and passen- ger satisfaction is lower than that of platform B and C levels. Since the immediate needs of passengers cannot be met, the loss of passengers will be caused, and the loss of passengers will further lead to the loss of drivers, resulting in a vicious cycle, and the platform's final revenue will be reduced.

Platform B adopts the dispatch rules; then the platform will use its complex algorithm to select one driver from 20 drivers to send an order with a competition ratio of 0 . The final matching result is determined by the algorithm itself and has a certain degree of controllability. The matching result is more in line with user expectations than platform A, but it is difficult to meet the immediate needs of passengers. Its user satisfaction is higher than that of platform A, and the matching time is no different than that of platform C. But user satisfaction is not as good as that of platform C.

Platform $\mathrm{C}$, which adopts the mechanism proposed in this article, will choose to send orders to 10 drivers from 20 drivers. The final competition ratio is determined by the number of single drivers but must be less than $1 / 10$. Driver's competition ratio is better than that of platform $\mathrm{A}$ and inferior to that of platform $\mathrm{B}$. However, users can choose the driver that best meets their needs among the $n$ drivers who grab the order. The user's immediate needs can be met to a certain extent, and user satisfaction will rise. The increase in user satisfaction will bring more new passengers to the platform, and the addition of new passengers will also bring more drivers. Ultimately, the platform's revenue will gradually increase as user satisfaction increases.

\section{Conclusion and Future Directions}

Inability to guarantee the retention rate of users and the amount of new users introduced are the problems that the ride-hailing platform needs to solve urgently. The key to solving this problem is to improve users' satisfaction and make the platform enter a virtuous cycle, thus increasing platform revenue. In order to solve this problem, this paper proposes an allocation mechanism. Through comparative experiments, it is proved that this mechanism can improve user satisfaction, make the platform enter a virtuous circle, obtain more benefits, and solve the problem of fairness selection under the existing matching rules.

However, this paper focuses on passenger satisfaction, and the users of the platform include not only passengers but also drivers. How to improve driver satisfaction will be our next step. At the same time, although the price function is introduced in this paper, it is not discussed further. How to develop a reasonable price function will also be the focus of the next research work.

\section{Data Availability}

The data used to support the findings of this study are included within the article.

\section{Conflicts of Interest}

The authors declare that there is no conflict of interest regarding the publication of this paper. 


\section{Acknowledgments}

This work was supported in part by the National Natural Science Foundation of China under Grant 61872313, in part by the Key Research Projects in Education Informatization in Jiangsu Province under Grant 20180012, in part by the Postgraduate Research and Practice Innovation Program of Jiangsu Province under Grant KYCX18 $\backslash 2366$, in part by the Yangzhou Science and Technology under Grant YZ2018209 and Grant YZ2019133, in part by the Yangzhou University Jiangdu High-End Equipment Engineering Technology Research Institute Open Project under Grant YDJD201707, and in part by the Open Project in the State Key Laboratory of Ocean Engineering, Shanghai Jiao Tong University, under Grant 1907.

\section{References}

[1] W. Chen, M. Mes, M. Schutten, and J. Quint, “A ride-sharing problem with meeting points and return restrictions," Transportation Science, vol. 53, no. 2, pp. 401-426, 2019.

[2] Y. Wang, J. Gu, S. Wang, and J. Wang, "Understanding consumers' willingness to use ride-sharing services: the roles of perceived value and perceived risk," Transportation Research Part C: Emerging Technologies, vol. 105, pp. 504-519, 2019.

[3] A. Henao and W. E. Marshall, "An analysis of the individual economics of ride-hailing drivers," Transportation Research Part A: Policy and Practice, vol. 130, pp. 440-451, 2019.

[4] J.-C. Yang and D.-D. Chen, "Influencing factors of customer satisfaction towards ride-sharing," Ecological Economy, vol. 15, no. 2, pp. 88-94, 2019.

[5] M. Gilibert, I. Ribas, N. Maslekar, C. Rosen, and A. Siebeneich, "Mapping of service deployment use cases and user requirements for an on-demand shared ride-hailing service: Moia test service case study," Case Studies on Transport Policy, vol. 7, no. 3, pp. 598-606, 2019.

[6] E. Segal-Halevi, A. Hassidim, and Y. Aumann, "Muda: a truthful multi-unit double-auction mechanism," in Thirty-Second AAAI Conference on Artifcial Intelligence, pp. 1193-1201, AAAI Press, New Orleans, 2018.

[7] D.-H. Lee, H. Wang, R. L. Cheu, and S. H. Teo, "Taxi dispatch system based on current demands and real-time traffic conditions," Transportation Research Record, vol. 1882, no. 1, pp. 193-200, 2004.

[8] F. Miao, S. Han, S. Lin et al., "Data-driven robust taxi dispatch under demand uncertainties," IEEE Transactions on Control Systems Technology, vol. 27, no. 1, pp. 175-191, 2019.

[9] C. Watanabe, K. Naveed, and P. Neittaanmäki, "Co-evolution of three mega-trends nurtures un-captured GDP - Uber's ride- sharing revolution," Technology in Society, vol. 46, pp. 164-185, 2016.

[10] Z. Xu, Z. Li, Q. Guan et al., "Large-scale order dispatch in ondemand ride-hailing platforms: a learning and planning approach," in Proceedings of the 24th ACM SIGKDD International Conference on Knowledge Discovery \& Data Mining, pp. 905-913, New York, 2018.

[11] A. Henao and W. E. Marshall, "The impact of ride-hailing on vehicle miles traveled," Transportation, vol. 46, no. 6, pp. 2173-2194, 2019.
[12] A. Pham, I. Dacosta, B. Jacot-Guillarmod et al., "PrivateRide: a privacy-enhanced ride-hailing service," Proceedings on Privacy Enhancing Technologies, vol. 2017, no. 2, pp. 38-56, 2017.

[13] M. Young and S. Farber, "The who, why, and when of uber and other ride-hailing trips: an examination of a large sample household travel survey," Transportation Research Part A: Policy and Practice, vol. 119, pp. 383-392, 2019.

[14] L. A. de Souza Silva, M. O. de Andrade, and M. L. Alves Maia, "How does the ride-hailing systems demand affect individual transport regulation?," Research in Transportation Economics, vol. 69, pp. 600-606, 2018.

[15] M. Mäntymäki, A. Baiyere, and A. K. M. N. Islam, "Digital platforms and the changing nature of physical work: Insights from ride-hailing," International Journal of Information Management, vol. 49, pp. 452-460, 2019.

[16] Y. Guo, X. Li, and X. Zeng, "Platform competition in the sharing economy: understanding how ride-hailing services influence new car purchases," Journal of Management Information Systems, vol. 36, no. 4, pp. 1043-1070, 2019.

[17] L. Ma, X. Zhang, X. Ding, and G. Wang, "Risk perception and intention to discontinue use of ride-hailing services in China: taking the example of DiDi Chuxing," Transportation Research Part F: Psychology and Behaviour, vol. 66, pp. 459-470, 2019.

[18] R. Lan, L. Sun, Z. Liu, H. Lu, C. Pang, and X. Luo, "MADNet: a fast and lightweight network for single-image super resolution," IEEE Transactions on Cybernetics, pp. 1-11, 2020.

[19] H. Lu, Y. Li, S. Mu, D. Wang, H. Kim, and S. Serikawa, "Motor anomaly detection for unmanned aerial vehicles using reinforcement learning," IEEE Internet of Things Journal, vol. 5, no. 4, pp. 2315-2322, 2018.

[20] H. Lu, Y. Li, M. Chen, H. Kim, and S. Serikawa, "Brain intelligence: go beyond artificial intelligence," Mobile Networks and Applications, vol. 23, no. 2, pp. 368-375, 2018.

[21] X. Xu, H. Lu, J. Song, Y. Yang, H. T. Shen, and X. Li, “Ternary adversarial networks with self-supervision for zero-shot crossmodal retrieval," IEEE Transactions on Cybernetics, vol. 50, no. 6, pp. 2400-2413, 2020.

[22] J. P. Dickerson, K. A. Sankararaman, A. Srinivasan, and P. Xu, "Allocation problems in ride-sharing platforms: online matching with ofine reusable resources," in Thirty-Second AAAI Conference on Artifcial Intelligence, pp. 1007-1014, AAAI Press, New Orleans, 2018. 